\title{
Characterization of Crude Oils through Alkyl Chain Based Separation by Gel Permeation Chromatography and Mass Spectrometry
}

Nadrah A. Alawani, Saroj K. Panda, Amal R. Lajami, Thunayyan A. Al-Qunaysi and Hendrik Muller*

Saudi Aramco, Research \& Development Center, Dhahran, 31311, Saudi Arabia

\section{Supporting Information}

Four Arabian crude oils, namely Arabian Extra Light (AXL), Light (AL), Medium (AM) and Heavy (AH), and one South American crude oil, Marlim (MB) were investigated in this study. Gel permeation chromatography (GPC) was used on the crude oils to produce fractions of reduced compositional complexity, followed by high resolution mass spectrometry using Fourier transform ion cyclotron resonance mass spectrometry (FT ICR-MS) analysis with atmospheric pressure photo ionization (APPI) on each fraction to provide a comprehensive chemical characterization of the aromatic compounds. Field desorption time of flight mass spectrometry (FD TOF MS) was also used to characterize the saturated compounds in the early eluting GPC fractions. 


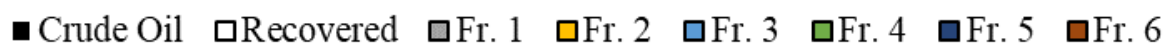

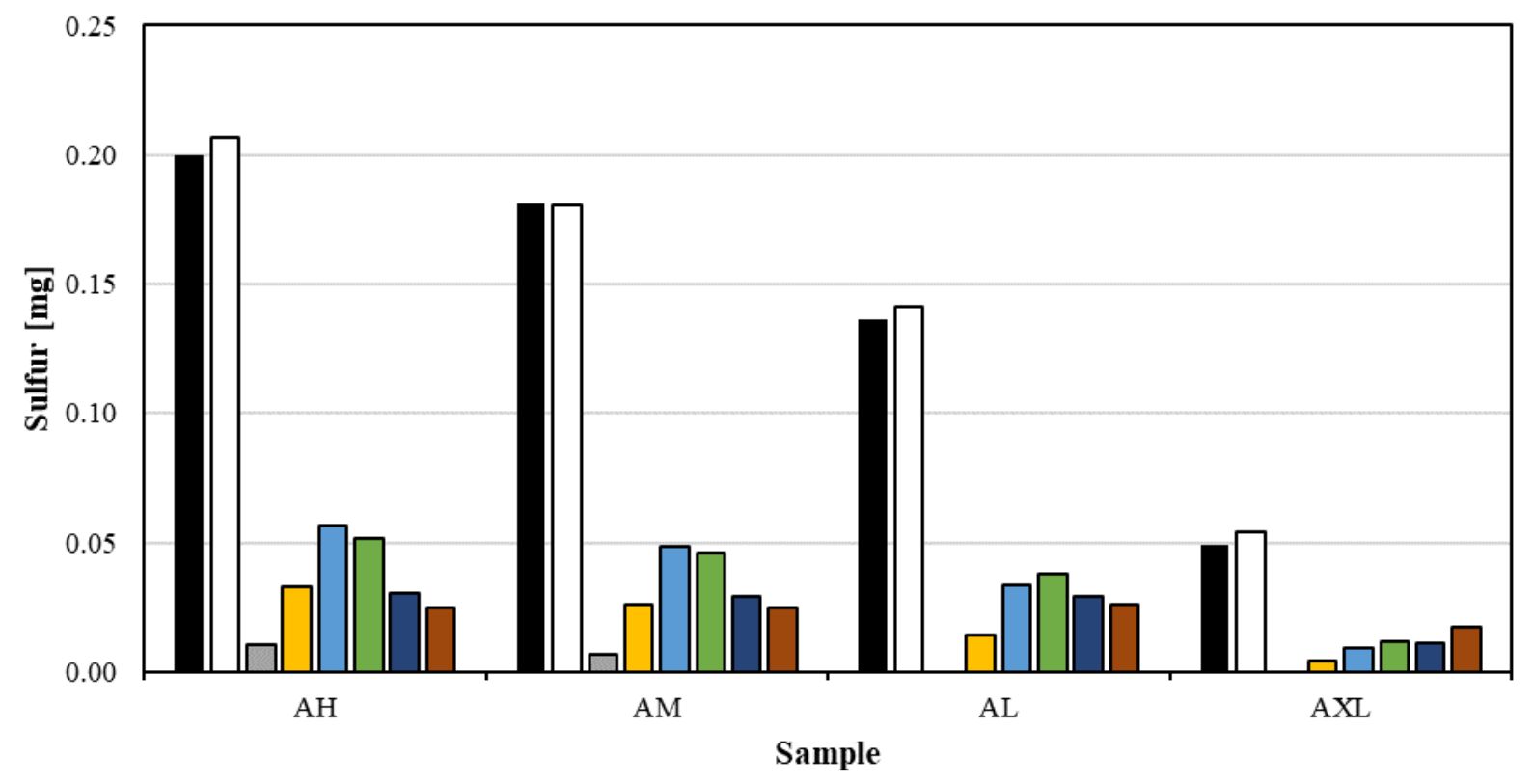

Figure S1. Absolut sulfur content of crude oil samples and GPC fractions. 
a)

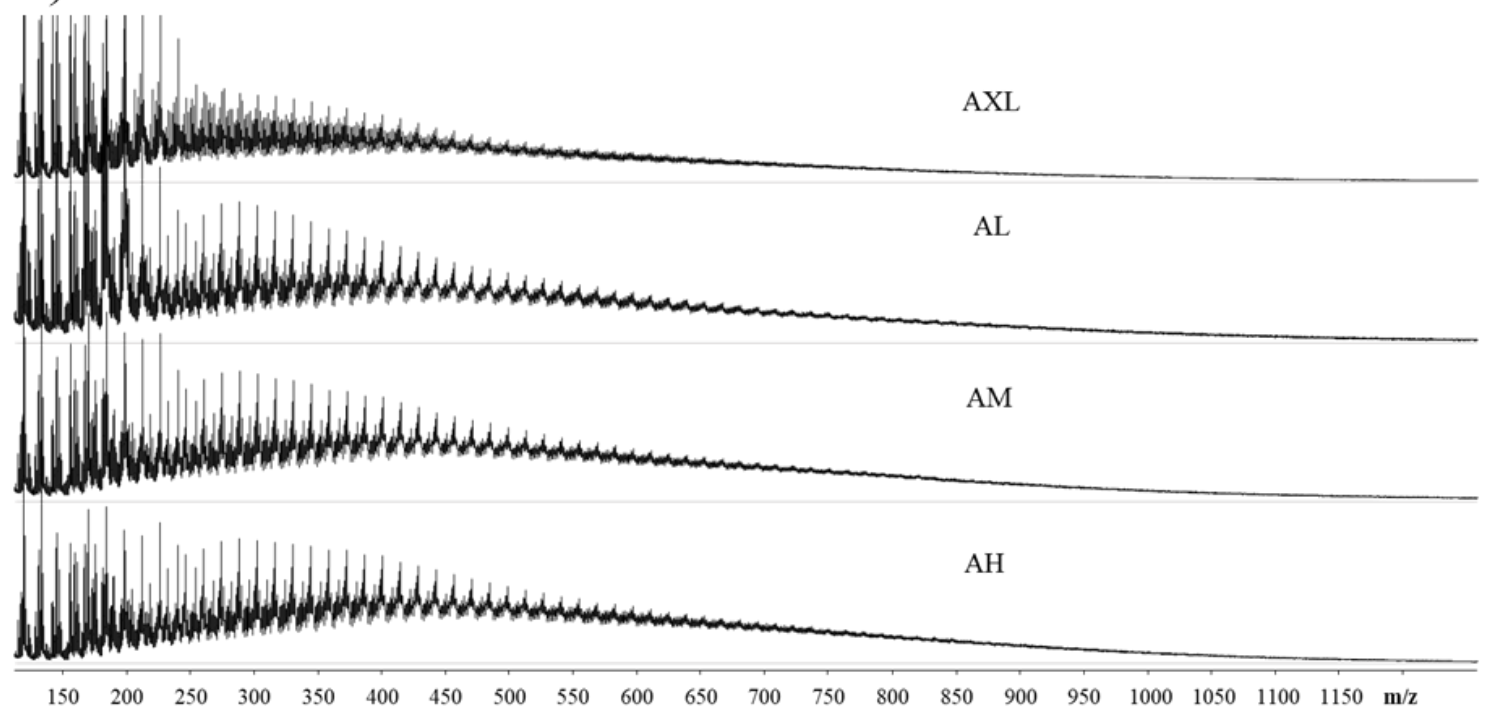

b)

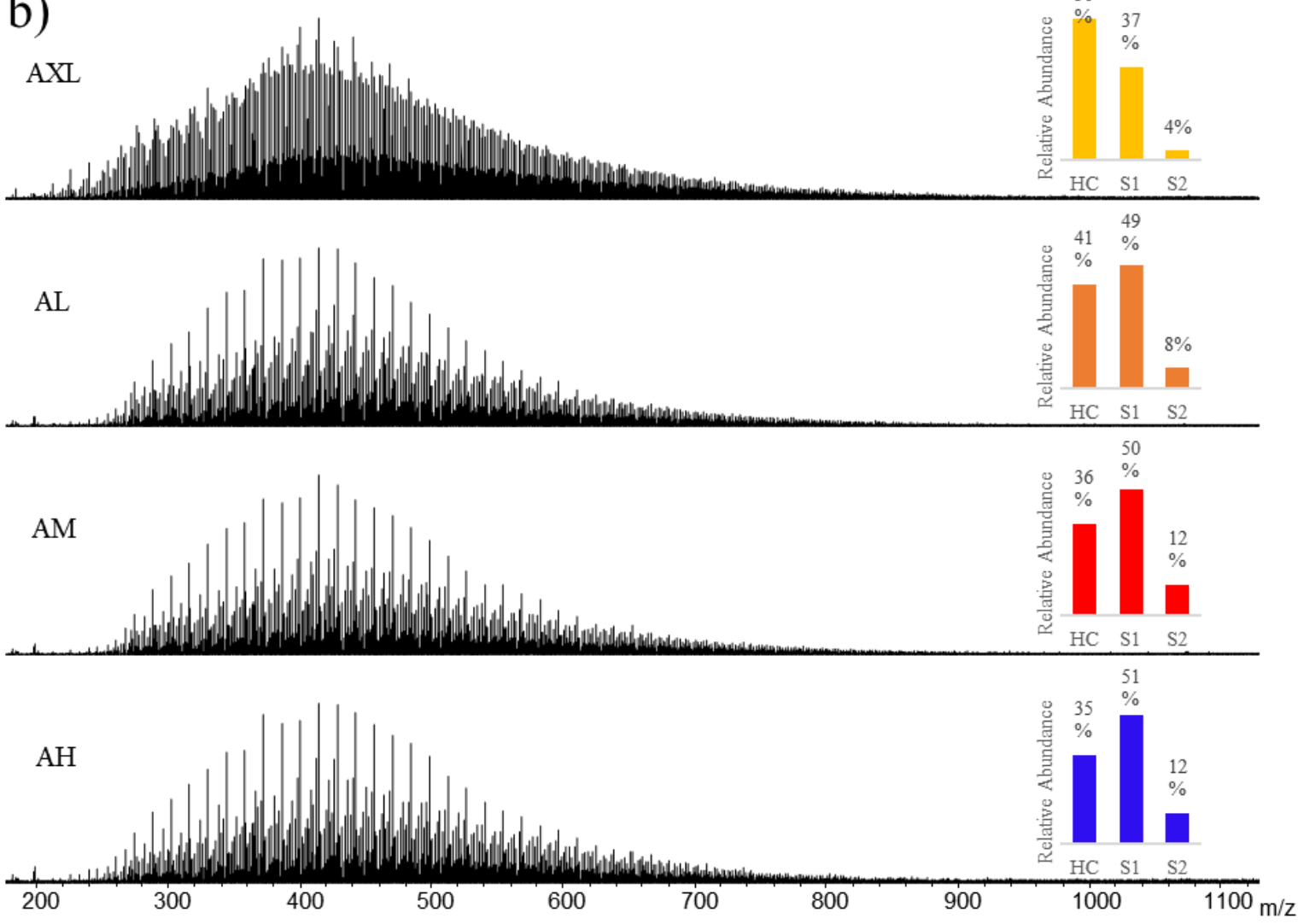

Figure S2. a) APPI time of flight mass spectra of AXL, AL, AM and AH crude oils showing their molecular weight distributions. b) APPI FT-ICR mass spectra of AXL, AL, AM and AH crude oils. The inserts on the right show the summed relative abundance of non-polar aromatic compound classes. 
AXI GPC fraction 2

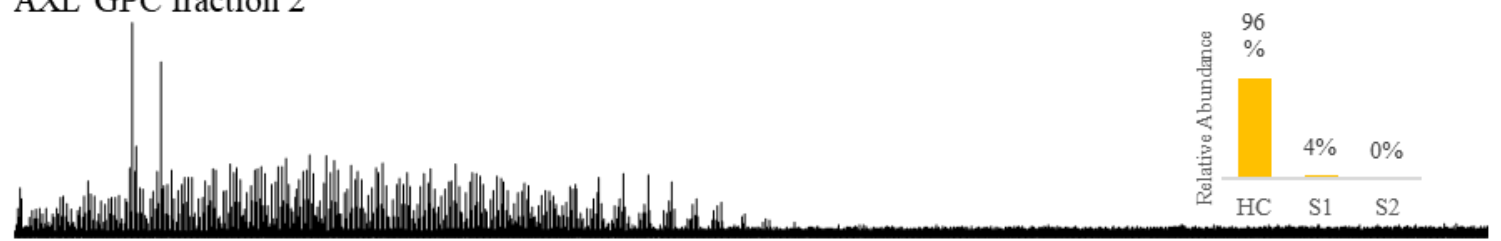

AL GPC fraction 2

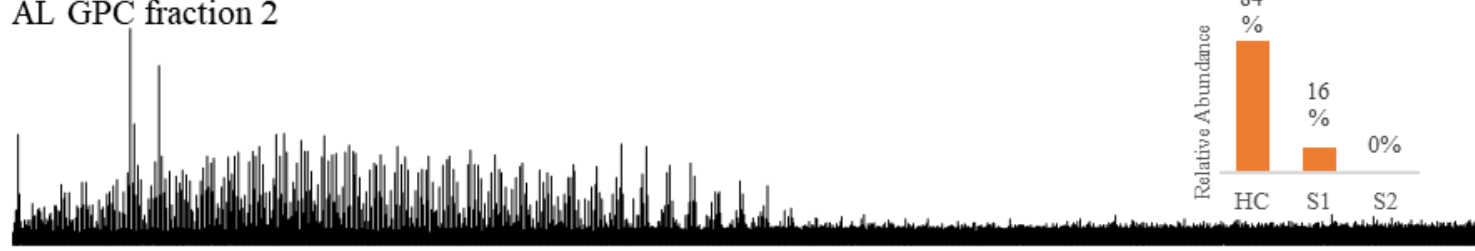

AM GPC fraction 2

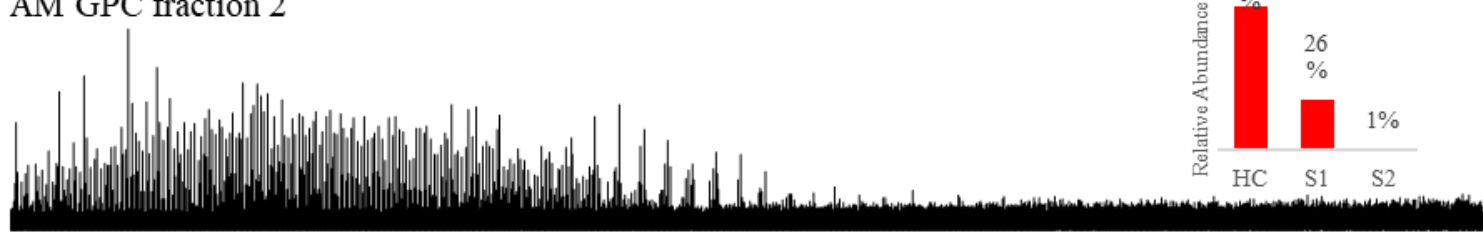

AH GPC fraction 2

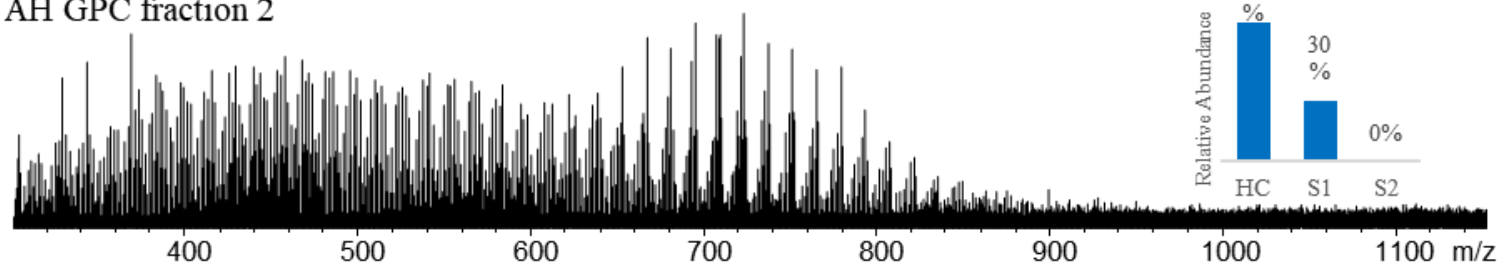

Figure S3. APPI FT-ICR mass spectra of GPC fraction 2 for AXL, AL, AM and AH crude oils. The inserts on the right show the summed relative abundance of main classes. 
AXI GPC fraction 3

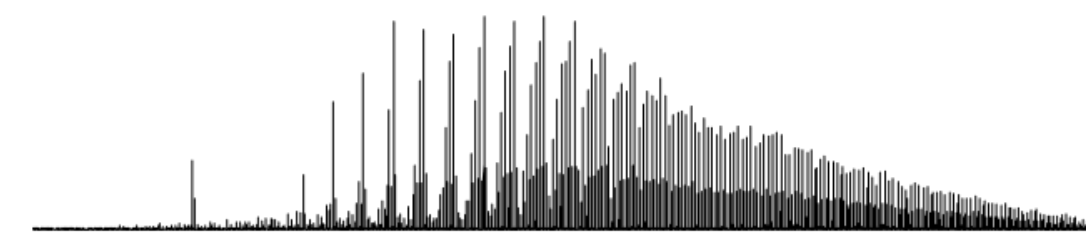

AL GPC fraction 3

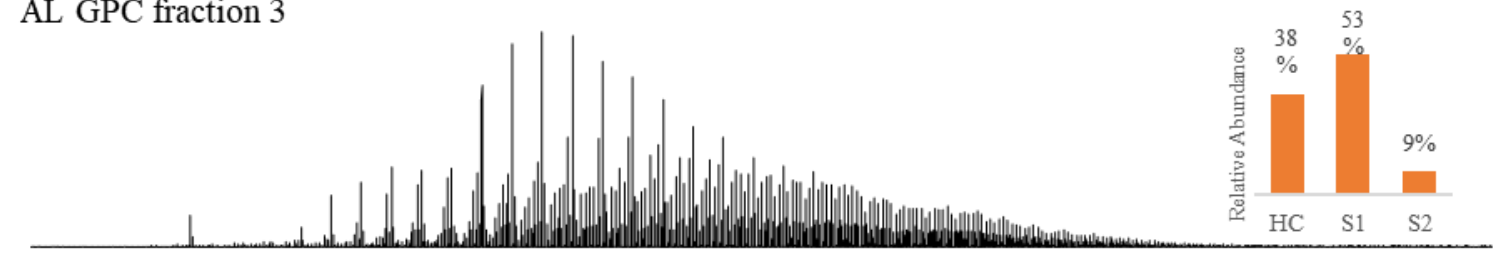

AM GPC fraction 3

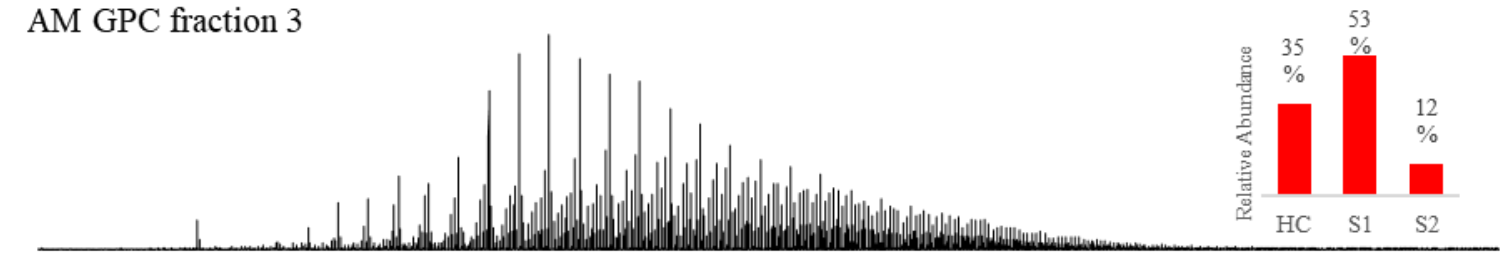

AH GPC fraction 3

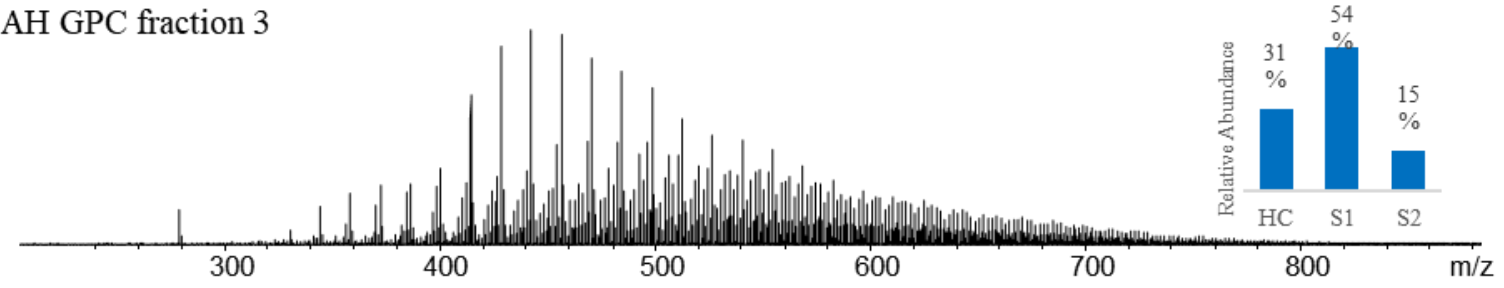

Figure S4. APPI FT-ICR mass spectra of GPC fraction 3 for AXL, AL, AM and AH crude oils. The inserts on the right show the summed relative abundance of main classes. 

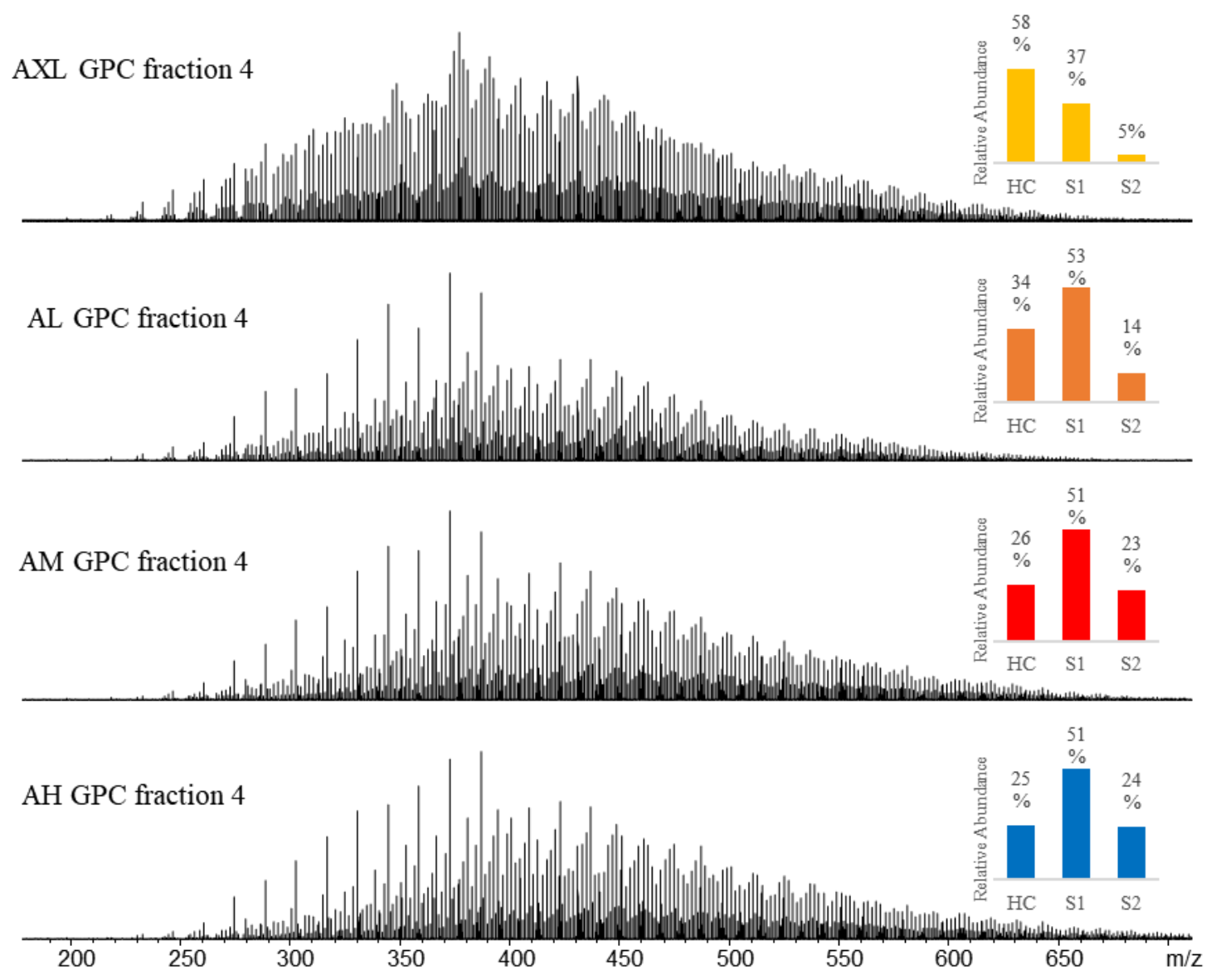

Figure S5. APPI FT-ICR mass spectra of GPC fraction 4 for AXL, AL, AM and AH crude oils. The inserts on the right show the summed relative abundance of main classes. 
AXI GPC fraction 5
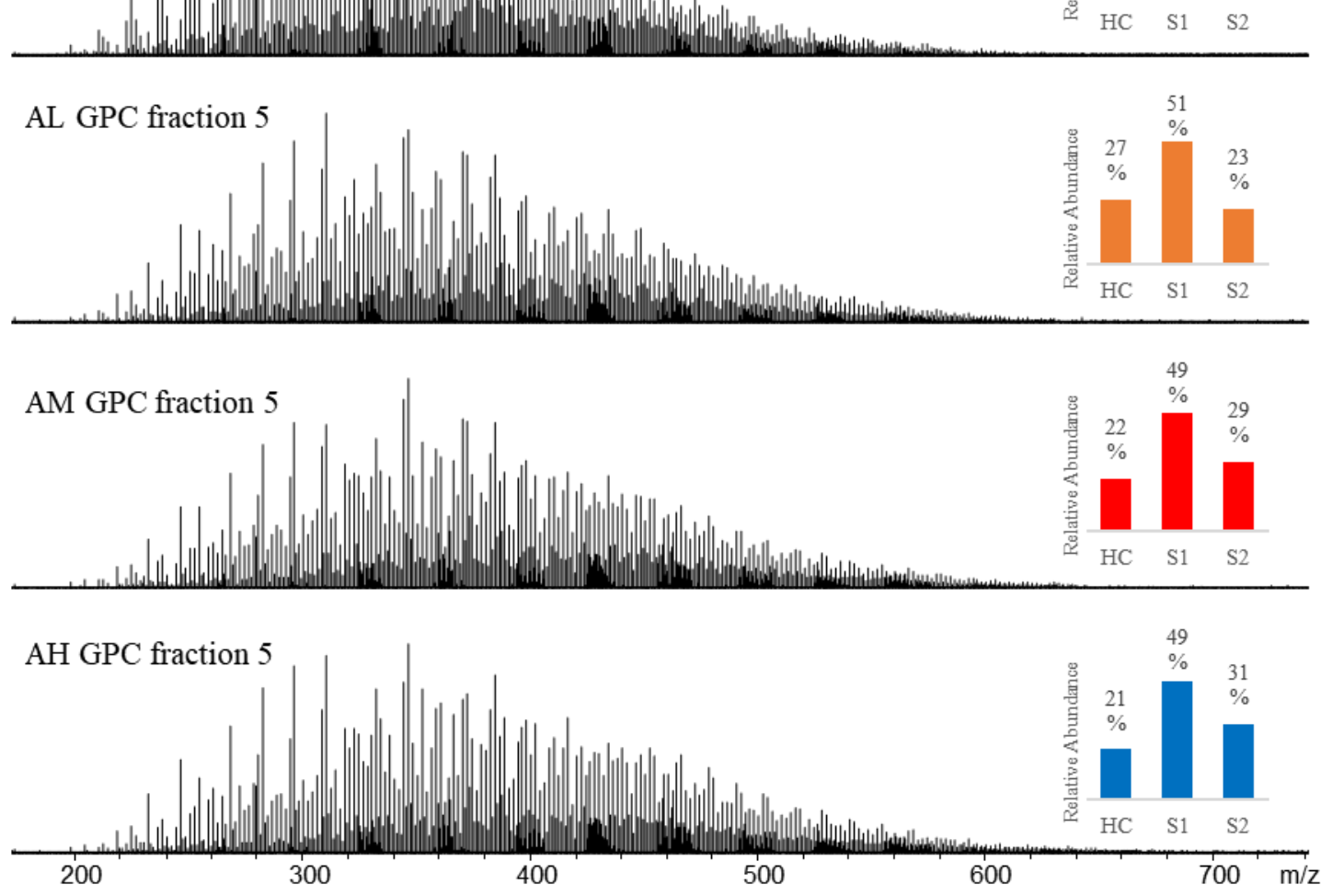

Figure S6. APPI FT-ICR mass spectra of GPC fraction 5 for AXL, AL, AM and AH crude oils. The inserts on the right show the summed relative abundance of main classes. 

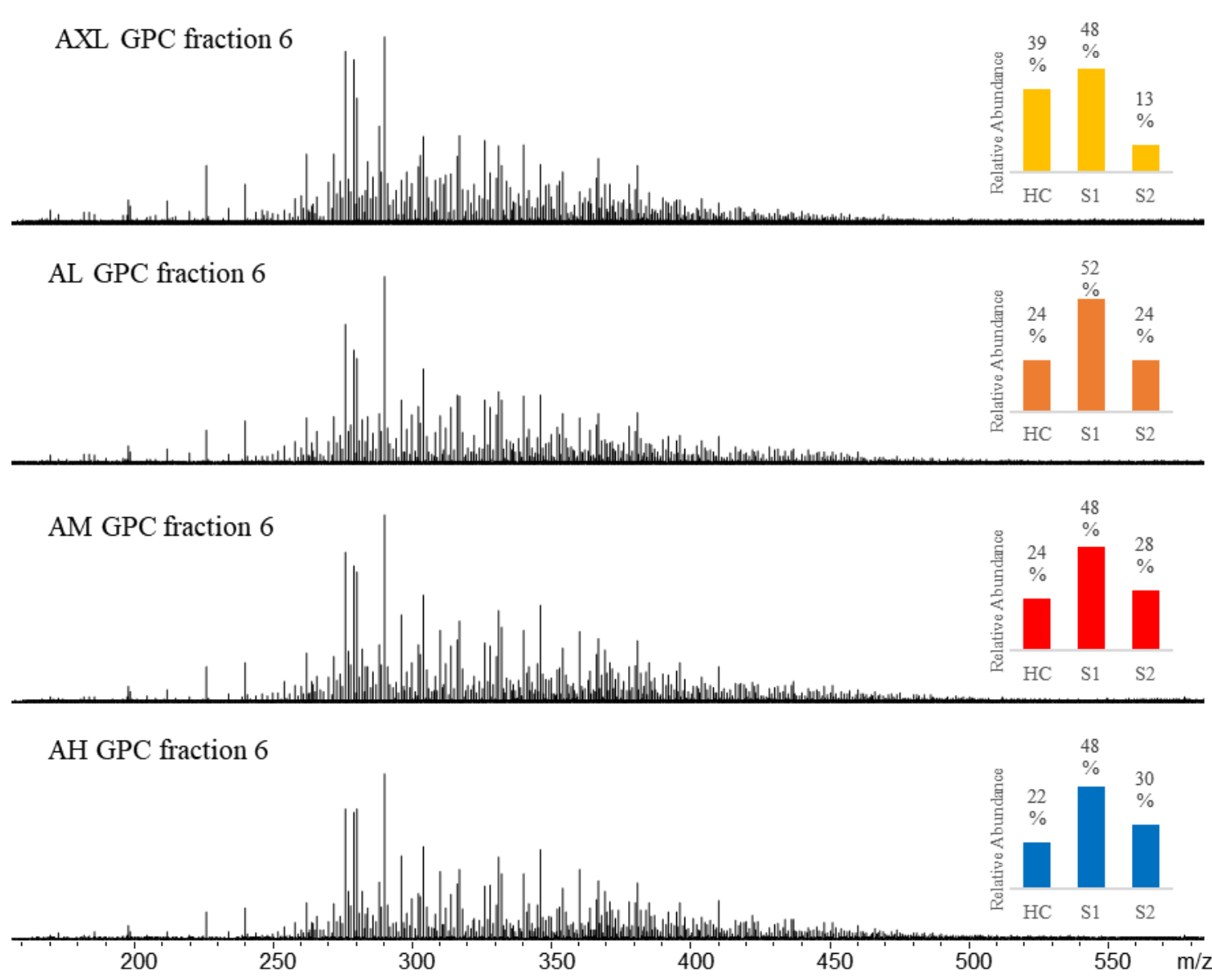

Figure S7. APPI FT-ICR mass spectra of GPC fraction 6 for AXL, AL, AM and AH crude oils. The inserts on the right show the summed relative abundance of main classes. 
Fragmentation pathways:

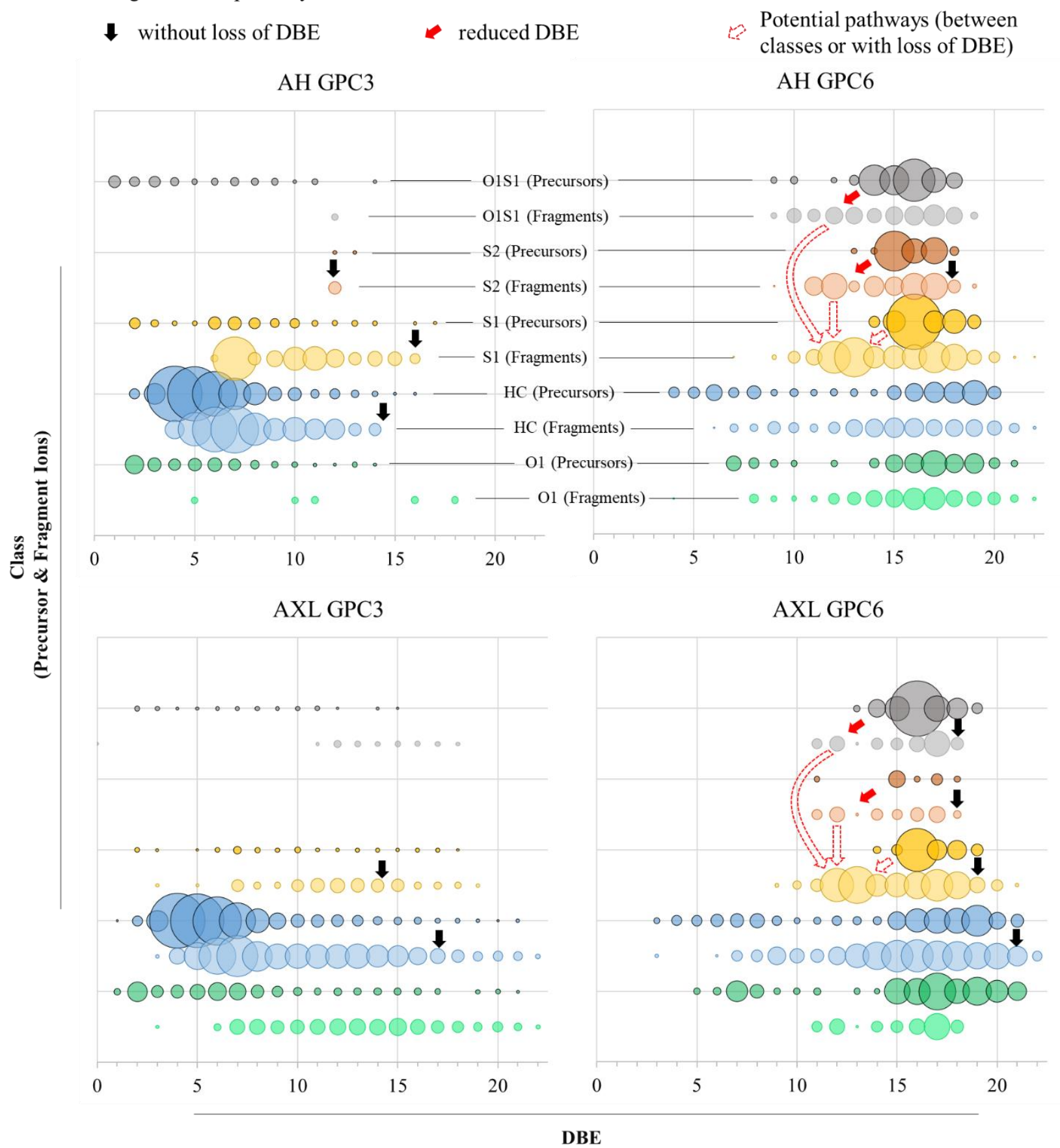

Figure S8. Overview of all classes' precursor and fragment ion DBE distributions after wide mass window isolation $(383 \pm 10 \mathrm{~m} / \mathrm{z}) \mathrm{CID}$ with argon gas at $20 \mathrm{~V}$. Some possible fragmentation pathways are indicated. 

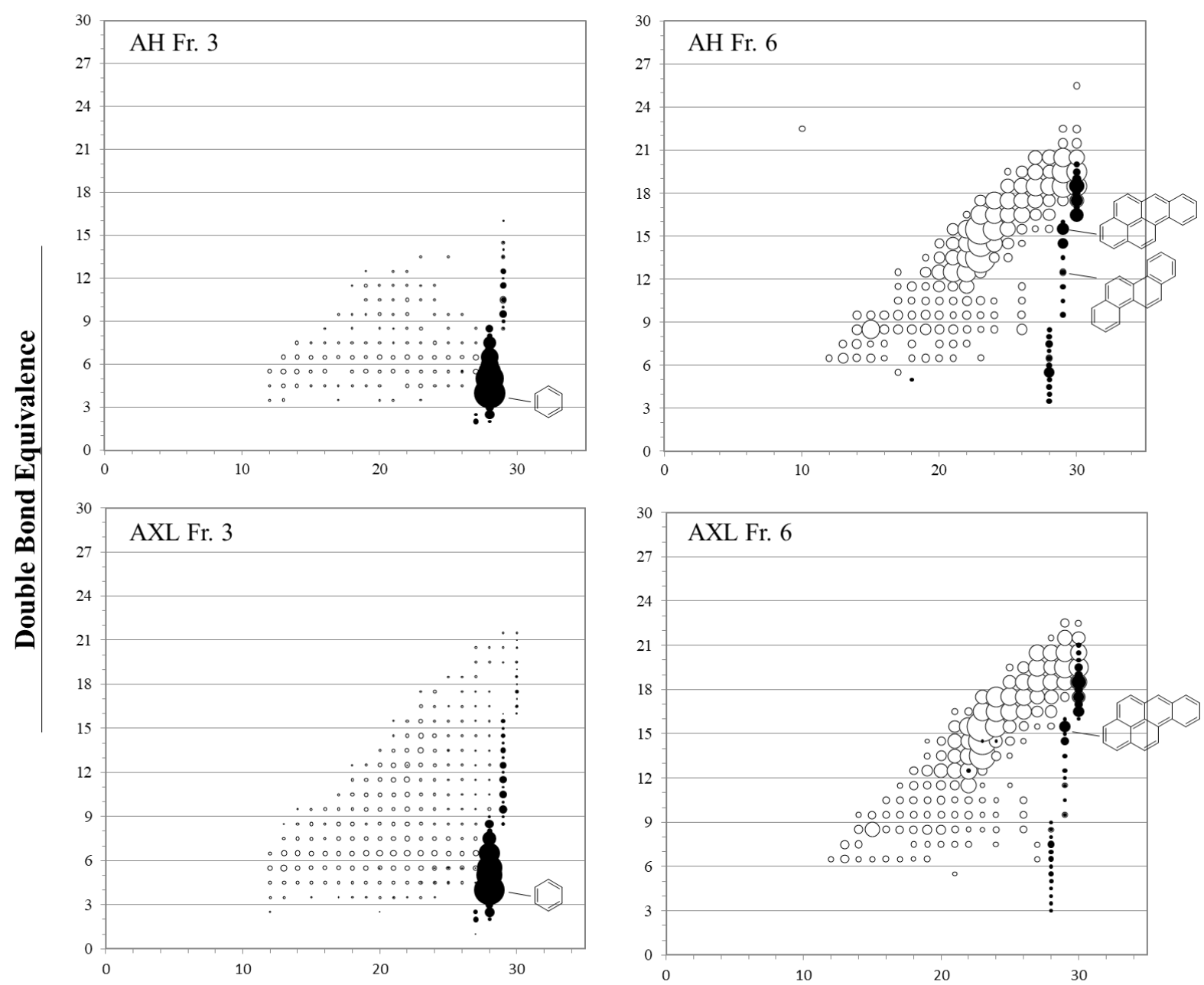

\section{Carbon Number}

Figure S9. Carbon number vs DBE plots of hydrocarbon (HC) class precursor $(\bullet)$ and fragment (०) ions after $\mathrm{m} / \mathrm{z}$ isolation $(383 \pm 10 \mathrm{~m} / \mathrm{z})$ and CID at $20 \mathrm{~V}$. Odd mass ions are plotted at the corresponding half-integer DBE value. Possible precursor ion structures (without alkyl side chains) are indicated. 

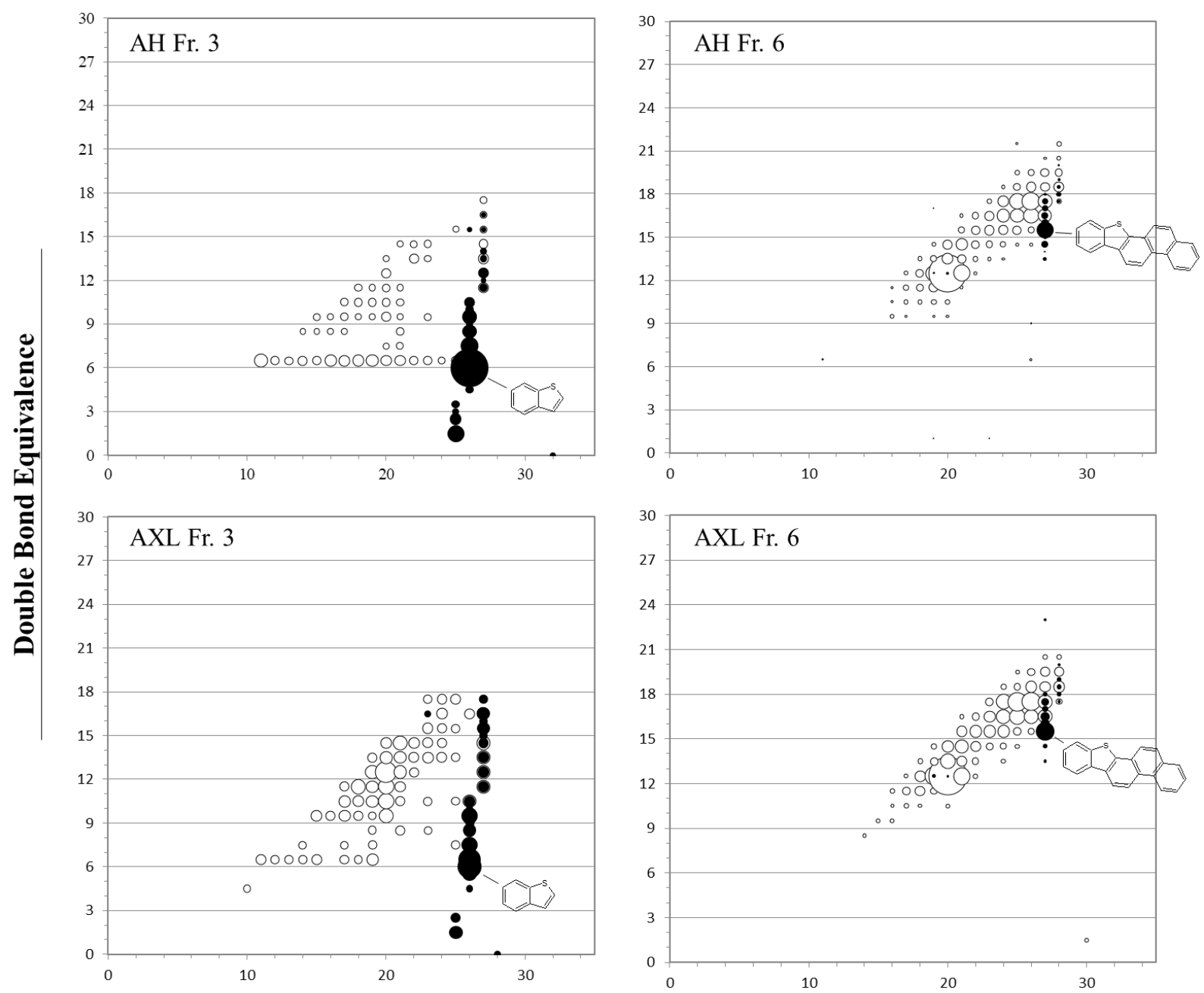

\section{Carbon Number}

Figure S10. Carbon number vs DBE plots of mono-sulfur (S1) class precursor $(\bullet)$ and fragment (०) ions after $\mathrm{m} / \mathrm{z}$ isolation $(383 \pm 10 \mathrm{~m} / \mathrm{z})$ and CID at $20 \mathrm{~V}$. Odd mass ions are plotted at the corresponding half-integer DBE value. Possible precursor ion structures (without alkyl side chains) are indicated. 

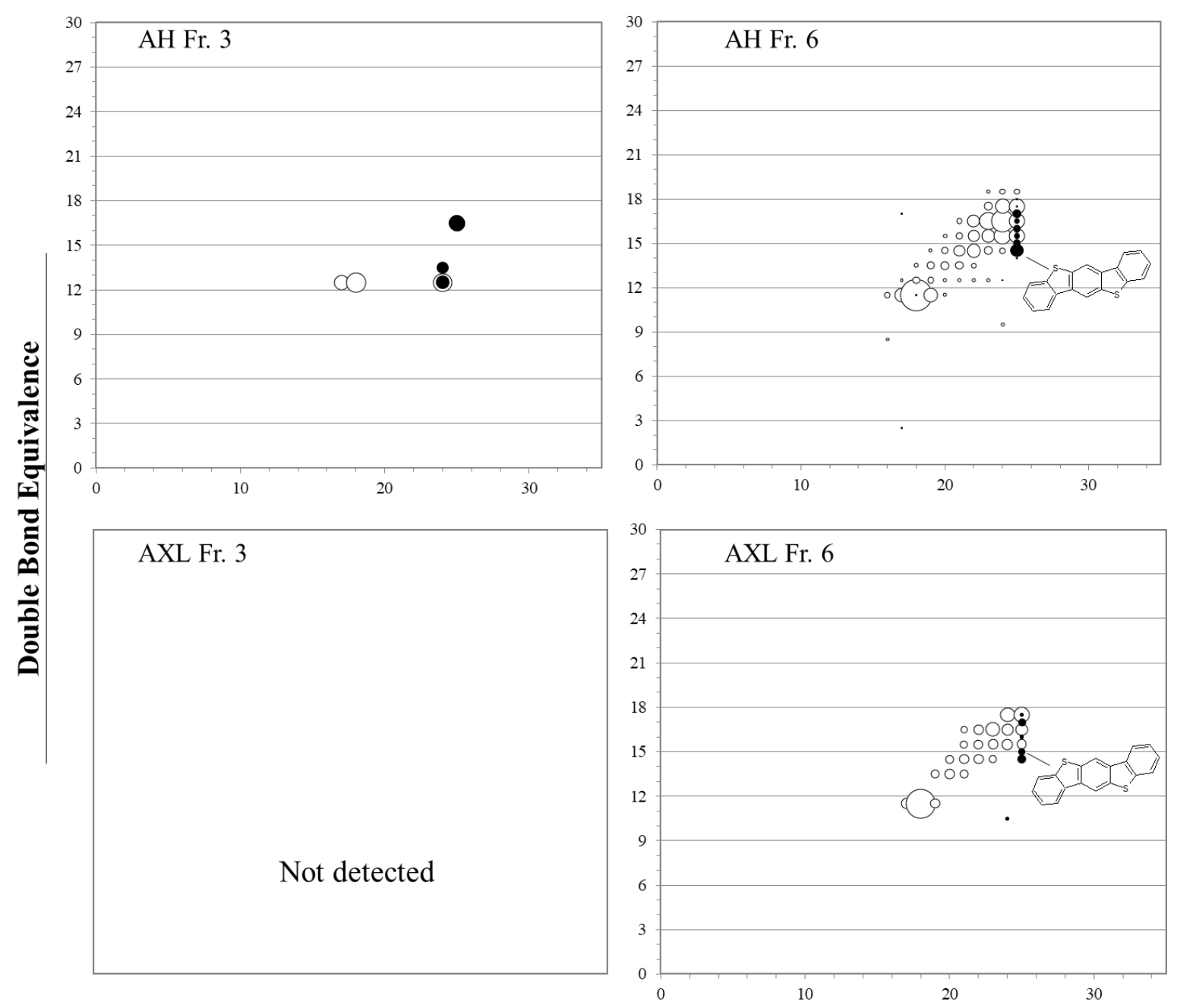

Carbon Number

Figure S11. Carbon number vs DBE plots of di-sulfur (S2) class precursor $(\bullet)$ and fragment (०) ions after $\mathrm{m} / \mathrm{z}$ isolation $(383 \pm 10 \mathrm{~m} / \mathrm{z})$ and CID at $20 \mathrm{~V}$. Odd mass ions are plotted at the corresponding half-integer DBE value. Possible precursor ion structures (without alkyl side chains) are indicated. 


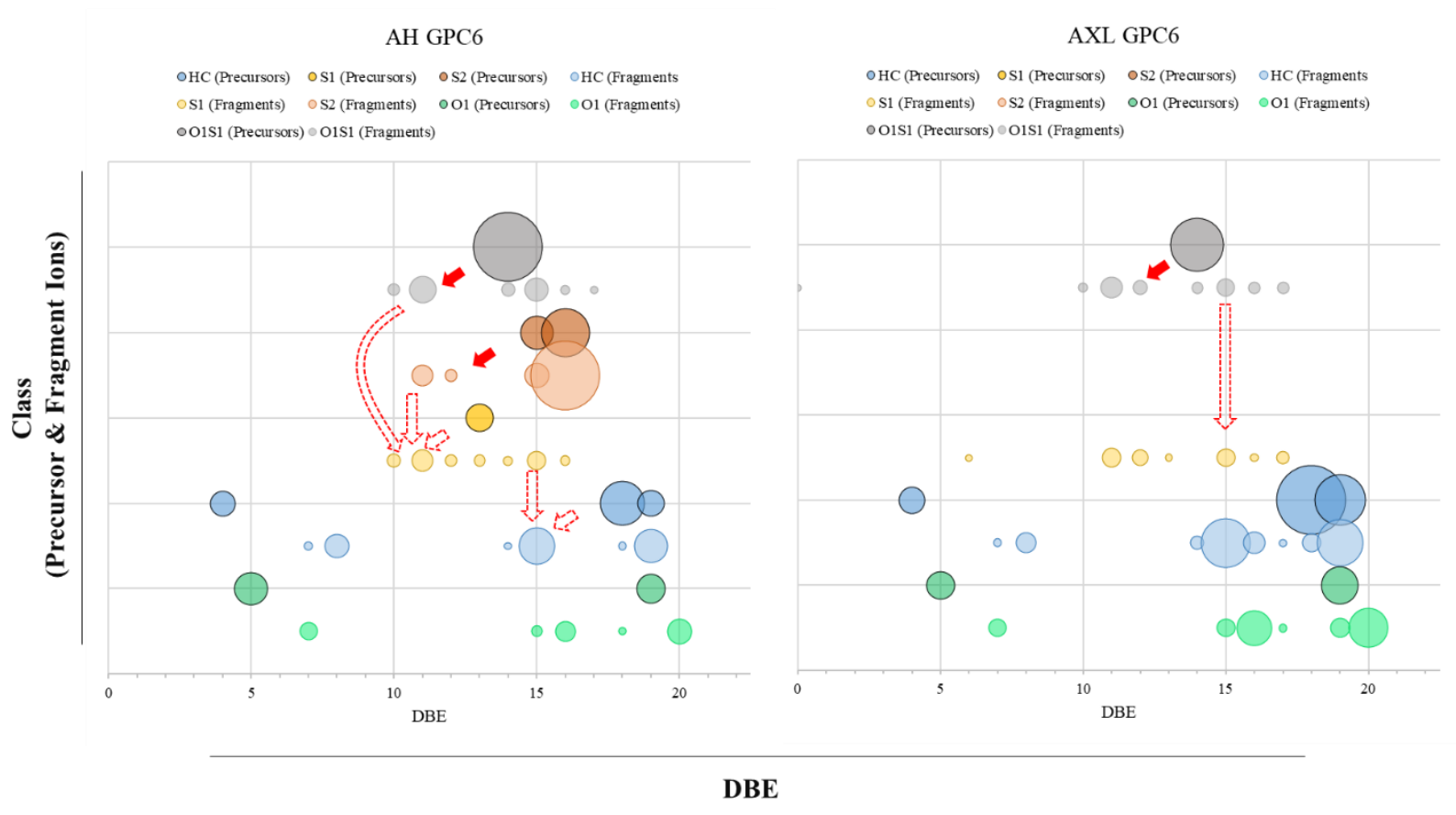

Figure S12. Precursor and fragment ion DBE distributions of the main observed classes after narrow mass window isolation $(385 \pm 1 \mathrm{~m} / \mathrm{z}$ ) CID with argon gas at $20 \mathrm{~V}$. Possible fragmentation pathways are indicated. Fragmentation entailing the loss of a non-condensed aromatic ring are indicated by red arrows. 


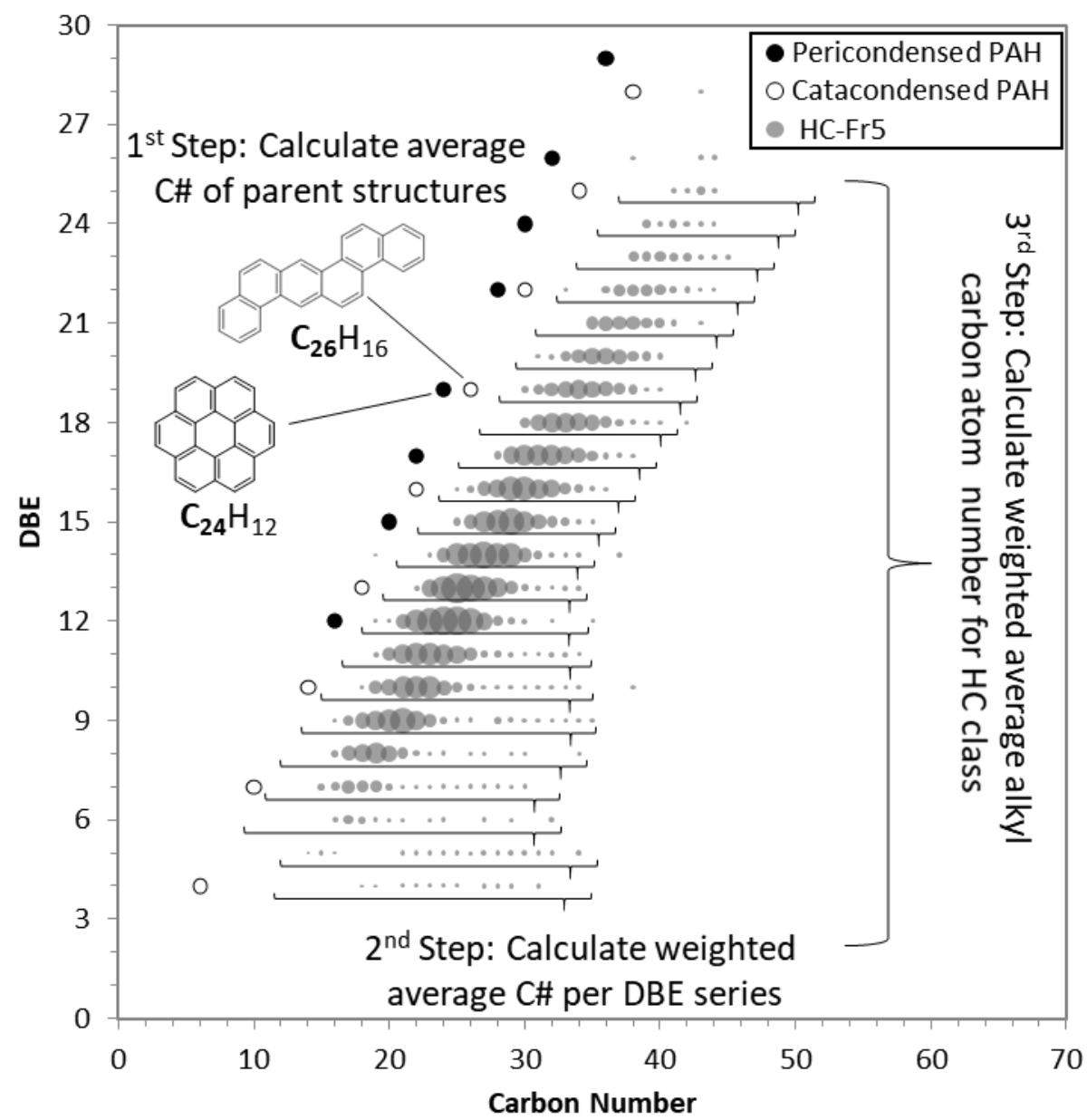

Figure S13. Graphic illustrating the calculation of the weighted average alkyl carbon atom number for the example of hydrocarbon species. $1^{\text {st }}$ the number of carbon atoms in the aromatic core structure is estimated as average between peri- and cata-condensed structures, $2^{\text {nd }}$ the weighted average carbon number is calculated for each DBE series, and subtraction of the corresponding aromatic core structure yields the alkyl carbon number for that series. $3^{\text {rd }}$ the weighted average is calculated for all DBE series, taking their relative summed abundances into account, to obtain the weighted average alkyl carbon number for the entire class. 